\title{
Apport d'un réseau de mesures des niveaux d'eau et de température à la compréhension de la dynamique côtière : Exemple du littoral de l'agglomération toulonnaise
}

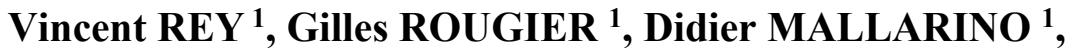 \\ Christiane DUFRESNE ${ }^{2}$, Isabelle TAUPIER-LETAGE ${ }^{1}$
}

1. Université de Toulon, CNRS/INSU, IRD, Mediterranean Institute of Oceanography (MIO), UM 110, 83041 Toulon Cedex 09, France.

Aix Marseille Université, CNRS/INSU, IRD, Mediterranean Institute of

Oceanography (MIO), UM 110, 13288 Marseille, France.

rey@univ-tln.fr

2. Ifremer, Laboratoire Environnement Ressource Provence-Azur-Corse, Station de

Bastia, Z.I. Furiani, Im. Agostini, 20600 Bastia, France.

\section{Résumé :}

Un réseau d'instruments de mesure a été installé dans différents ports de la côte varoise, entre les îles d'Hyères à l'est et la baie de Sanary à l'ouest. Deux capteurs piézométriques, l'un immergé et l'autre émergé, permettent de connaitre la pression atmosphérique, le niveau d'eau et la température. Les données de niveau permettent de retrouver les signaux de marée, semi-diurnes ou diurnes, d'amplitude environ $0.10 \mathrm{~m}$, et font apparaitre de plus fortes variations de niveau, de l'ordre de $1 \mathrm{~m}$, associées aux variations barométriques. A l'échelle de la petite rade de Toulon, des seiches apparaissent à plusieurs périodes de quelques dizaines de minutes. Les amplitudes observées aux différents points de mesure permettent d'appréhender leurs caractéristiques. Les évolutions des températures, associées notamment à des remontées d'eaux froides (ou upwellings) permettent d'appréhender la circulation des masses d'eau à l'échelle des baies.

Mots-clés : Réseau d'observation, Niveaux d'eau, Température, Marée, Surcote, Seiche, Upwelling.

\section{Introduction}

Le littoral de l'agglomération toulonnaise et plus largement de la côte varoise présente des caractéristiques très variées (plages sableuses, côtes rocheuses, bathymétries fortement variables, installations et bassins portuaires, archipels), une exposition diverse aux tempêtes et une forte anthropisation. Le but du réseau d'observation HTM-NET est d'apporter des informations originales continues et pérennes de la dynamique par la mesure des niveaux d'eau et des températures près de la surface en différents points de la zone, pour une meilleure compréhension et modélisation des interactions et couplages 


\section{Thème 7 - Risques côtiers}

entre la dynamique à l'échelle côtière et à l'échelle des baies de l'agglomération toulonnaise.

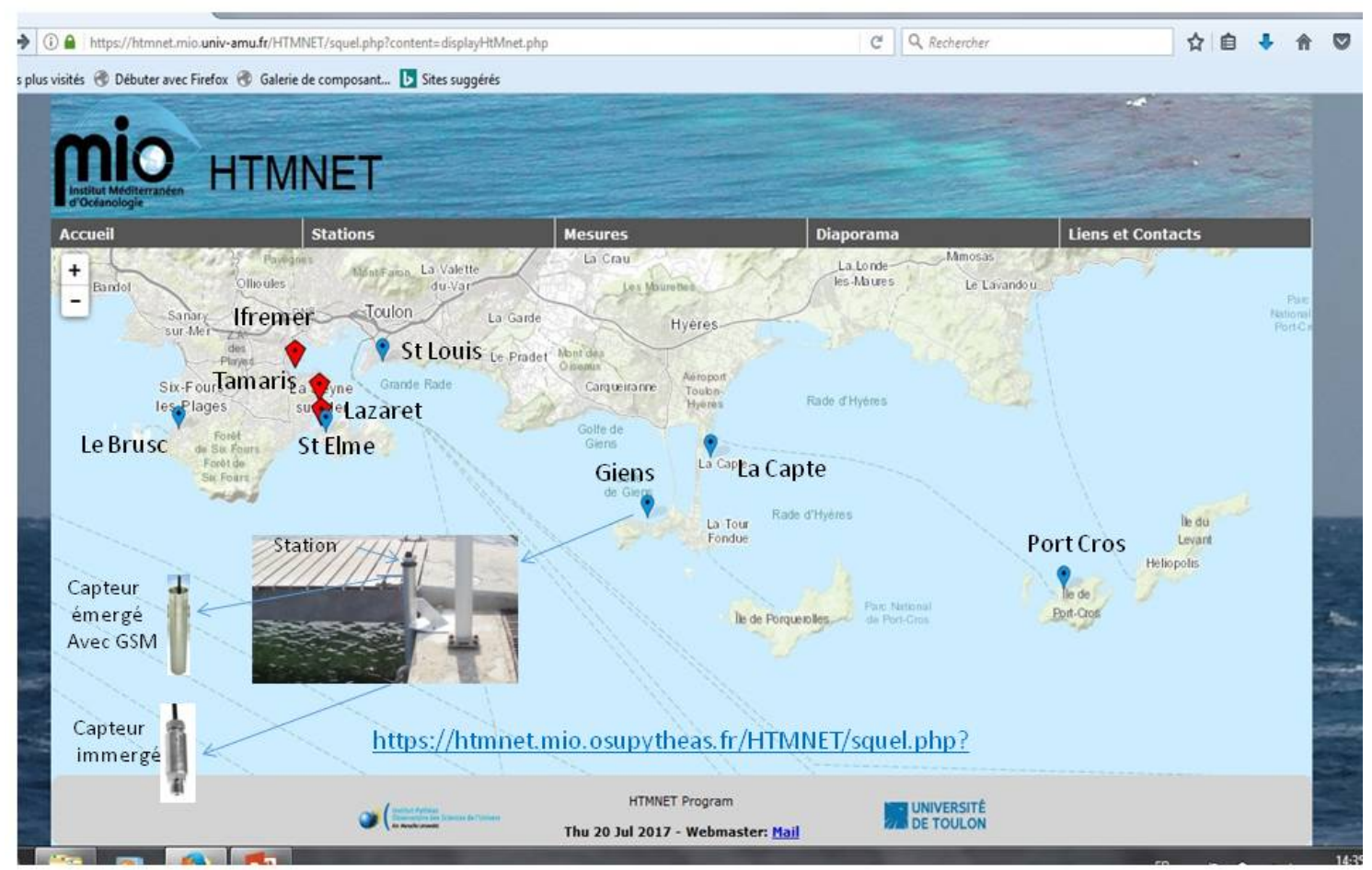

Figure 1. Implantation des instruments.

La circulation générale le long du littoral varois est forcée par le Courant Nord (ou Liguro-Provençal), d'un débit d'environ 1 millions de $\mathrm{m}^{3} / \mathrm{s}$, qui longe la côte depuis la mer Ligure puis présente une dynamique plus complexe à partir des îles d'Hyères, à cause de la bathymétrie complexe, puis à l'ouest de Toulon où débute le plateau continental du Golfe du Lion (GUIHOU, 2013; TAUPIER-LETAGE et al., 2003). C'est également une zone soumise à deux vents dominants, le Mistral, d'ouest à nordouest et le vent d'est. Le vent d'est correspond généralement à des épisodes dépressionnaires, contrairement au Mistral. L'orientation de la côte par rapport au Mistral est à l'origine de remontées d'eaux froides (ou upwellings), et de mouvements de masses d'eaux importants à la côte (MILLOT, 1979).

Nous présentons ici le réseau de mesures et des résultats typiques sur les niveaux d'eau et la circulation qu'il permet d'appréhender.

\section{Le réseau d'observation}

Un réseau d'instruments de mesure a été installé dans différents ports de la côte, entre les îles d'Hyères à l'est et la baie de Sanary à l'ouest (voir Fig. 1). Deux capteurs 


\section{XVèmes Journées Nationales Génie Côtier - Génie Civil La Rochelle, 29 au 31 mai 2018}

piézométriques de la société Keller, l'un immergé et l'autre émergé, sont disposés à l'intérieur d'un tube cylindrique en aluminium, fixé verticalement sur des quais ou pontons. Ils permettent de connaitre la pression atmosphérique, le niveau d'eau et la température au niveau des instruments. Le niveau d'eau $\eta$ est égal à $\Delta p / \rho g$ avec $\Delta p$ la différence de pression dans l'air et dans l'eau, $\rho$ la masse volumique de l'eau fixée à 1.025 (valeur pour une salinité $\mathrm{S}=35$ et une température $\mathrm{T}=19^{\circ} \mathrm{C}$ ), $\mathrm{g}=9.81$ l'accélération due à la gravité, les variations de pression dans la colonne d'air sont négligées. Actuellement, le réseau est composé de 9 instruments autonomes dont 6 munis d'un GSM (voir Tab. 1, et signalés en bleu sur la Fig. 1), qui permettent un suivi en temps légèrement décalé (quelques heures) des données acquises toutes les dix minutes (consultables sur le site https://htmnet.mio.osupytheas.fr/HTMNET/squel.php?content=accueil.php).

La précision de la hauteur d'eau est d'environ $1 \mathrm{~mm}$, celle de la température de 0.5 degrés. Les erreurs de température ou de pression associées à la salissure biologiques (biofouling) restent faibles mais des mesures ponctuelles bimestrielles de référence de niveau d'eau et de température ont été initiées fin 2007 pour corriger les éventuelles dérives électroniques des capteurs. Elles sont accompagnées pour le niveau d'eau par un référencement des stations par rapport au niveau NGF en cours mené par le Shom, les données de niveau du réseau HTM-NET sont également accessibles sur le site REFMAR du Shom.

A titre d'exemple, les données de niveau d'eau (par rapport au zéro NGF) et de température de la station d'Ifremer sont présentées sur la figure 2.

Tableau. 1. Sites et dates d'installation.

\begin{tabular}{ll}
\hline Site & Date d'installation \\
\hline Ifremer & 17 octobre 2013 (prototype) \\
Port-Cros & $1^{\text {er juillet } 2014}$ \\
Lazaret & 13 mars 2015 \\
Tamaris & 18 mars 2015 \\
Madrague de Giens (transmission GSM) & 6 mai 2015 \\
Port Saint Louis du Mourillon (GSM) & 18 décembre 2015 \\
Port de saint Elme (GSM) & 12 février 2016 \\
Port du Brusc (GSM) & 20 avril 2016 \\
Port de La Capte (GSM) & 6 avril 2017 \\
\hline
\end{tabular}




\section{Thème 7 - Risques côtiers}
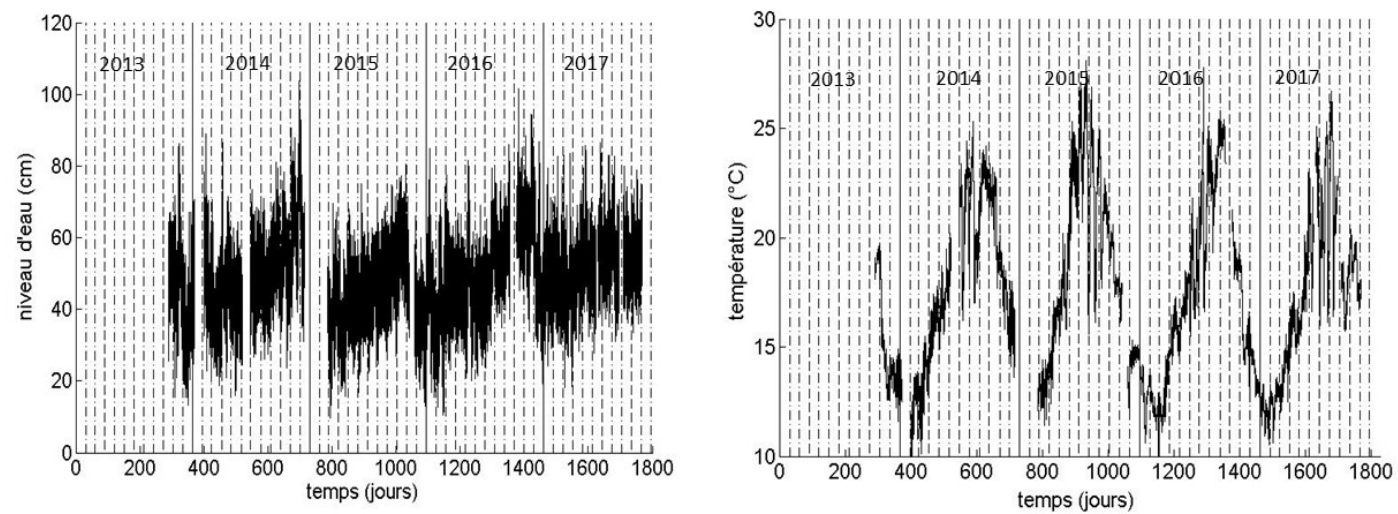

Figure 2. Exemple de série de données : site Ifremer.

\section{Analyse des variations de niveaux d'eau}

3.1 Marées, surcotes et décotes

Les séries temporelles de niveaux d'eau des différentes stations présentent des oscillations rapides des niveaux d'eau dues à la marée, et des variations plus lentes mais d'amplitude significative associées aux conditions météorologiques (notamment barométriques).
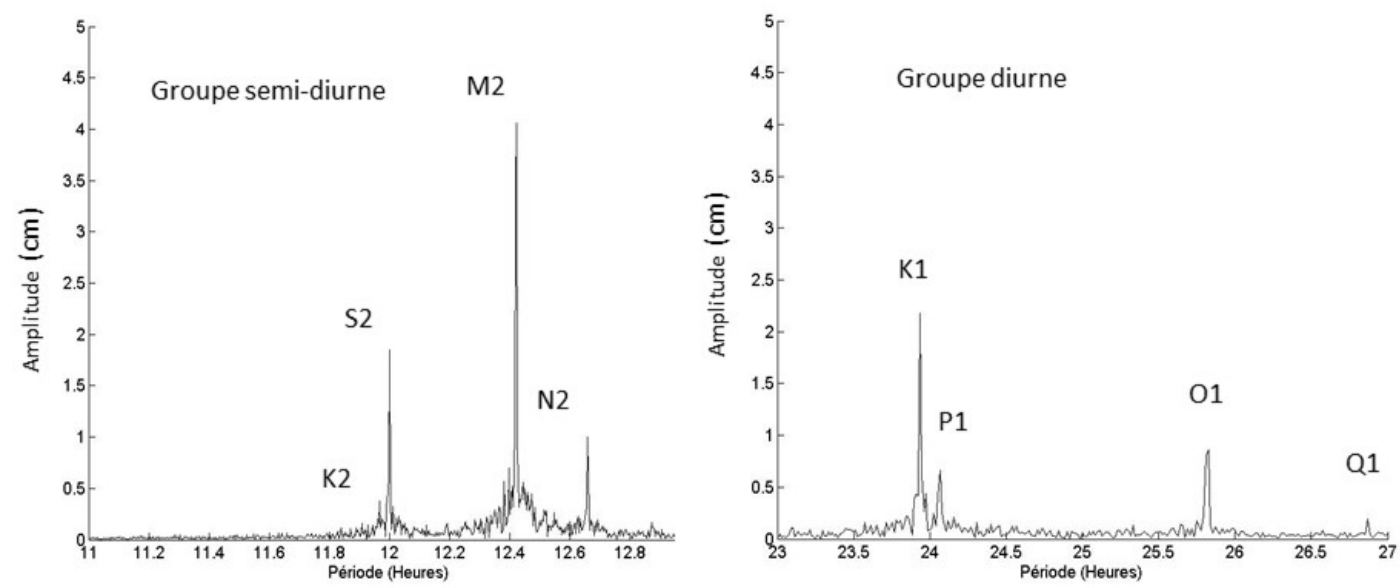

Figure 3. Analyse spectrale des données du site Ifremer.

L'analyse spectrale du signal pour la station Ifremer (voir figure 2) sur toute la durée des mesures (les données manquantes sont remplacées par la dernière valeur mesurée précédemment) permet d'identifier les composantes harmoniques de la marée les plus significatives. On peut observer sur la figure 3 l'identification des composantes K2, S2, M2, N2 pour le groupe semi-diurne et des composantes K1, P1, O1, Q1 pour le groupe 


\section{XVèmes Journées Nationales Génie Côtier - Génie Civil La Rochelle, 29 au 31 mai 2018}

diurne. Les amplitudes précises de ces composantes sont alors obtenues à partir d'une série temporelle suffisamment longue et complète, par le calcul des coefficients de Fourier (donc amplitude et phase) de chacune des périodes (connues exactement) composant le signal de marée. Les coefficients calculés à partir du traitement des séries temporelles du 8 septembre 2016 au 30 octobre 2017 pour les stations d'Ifremer et de Port Cros sont présentés dans le Tab. 2. On observe que les amplitudes sont voisines sur les deux sites et que le marnage maximal est de l'ordre de $20 \mathrm{~cm}$ (composantes S2, M2 et $\mathrm{K} 1$ en phase).

Tableau. 2. Composantes de marée.

\begin{tabular}{|c|c|c|}
\hline Composante de marée & amplitude (en cm), Ifremer & amplitude (en cm), Port-Cros \\
\hline$K 2(T=11.967 h)$ & 0.71 & 0.38 \\
\hline$S 2(T=12.00 h)$ & 2.66 & 1.95 \\
\hline M2 $(T=12.4206 h)$ & 4.11 & 5.26 \\
\hline N2 $(T=12.658 h)$ & 1.52 & 1.19 \\
\hline$K 1(T=23.935 h$ & 2.95 & 2.98 \\
\hline$P 1(T=24.066 h)$ & 0.93 & 0.79 \\
\hline O1 $(T=25.891 h)$ & 0.24 & 0.28 \\
\hline Q1 $(T=26.87 \mathrm{~h})$ & 0.32 & 0.23 \\
\hline
\end{tabular}
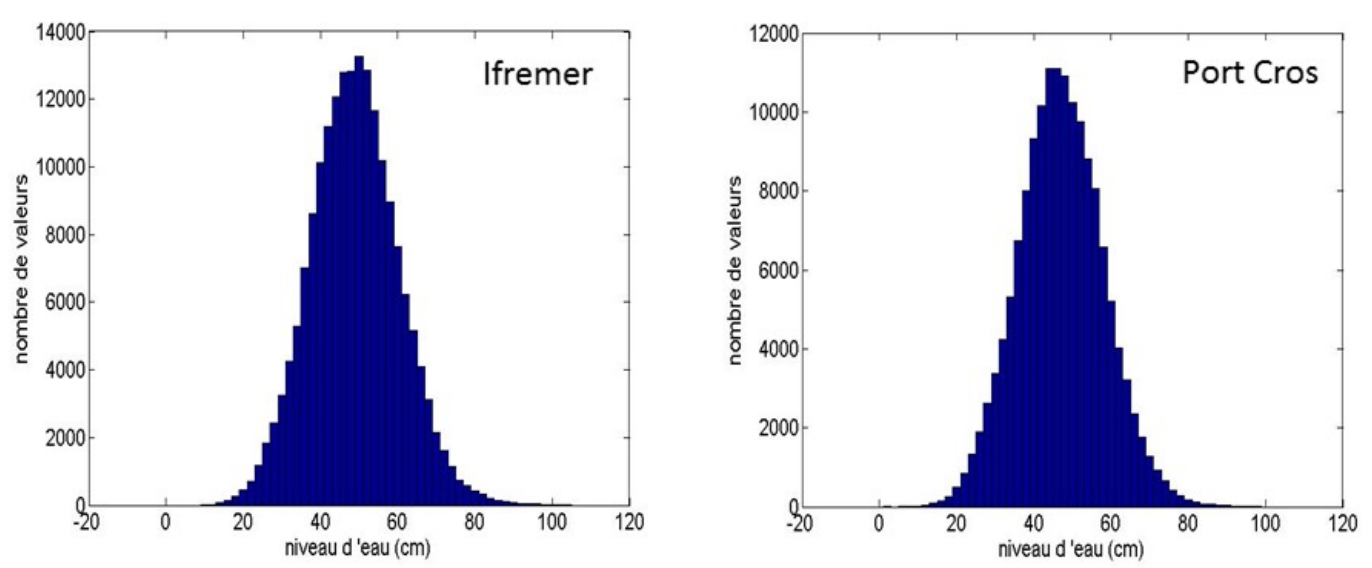

Figure 4. Histogramme des hauteurs d'eau.

Nous avons représenté sur la figure 4 l'histogramme des niveaux mesurés, exprimés par rapport au zéro NGF, sur les sites d'Ifremer et de Port Cros à partir de l'ensemble des enregistrements. On observe des variations de niveau de l'ordre d'un mètre, nettement supérieures à celles associées au marnage, et en grande partie dues aux variations barométriques. Des calculs d'analyse (non présentés ici) d'anomalie de niveau, c'est-à- 


\section{Thème 7 - Risques côtiers}

dire de différence entre le niveau (après filtrage de la marée) et l'opposé de la variation de niveau correspondant aux variations barométriques montrent que le niveau d'eau suit généralement à peu près l'évolution des conditions barométriques (ce qui correspond à l'hypothèse isostatique), bien que des anomalies supérieures à une vingtaine de centimètres soient parfois observées.

\subsection{Seiches}

D'autres sources de variations de hauteurs d'eaux sont les seiches, généralement observées dans des bassins fermés ou semi-fermés comme les ports ou les baies. Une étude de MILLOT et al (1981) a montré la présence de telles oscillations dans la rade de Toulon, en particulier à des périodes de $20 \mathrm{~min}$ et 45 minutes. La seiche de période 20 min est attribuée à une seiche en bassin fermé dans la petite rade présentant une nodale perpendiculaire à la grande jetée qui sépare petite et grande rades, tandis que celle de période 45 minutes correspond au $1^{\mathrm{er}}$ mode de l'ensemble petite rade plus grande rade. Les spectres issus de nos séries de données des stations dans la rade de Toulon (non montrés ici) font apparaitre des pics diffus autour de 45 minutes et près de $20 \mathrm{~min}$, sachant que cette dernière valeur est à la limite du sous-échantillonnage, la période de nos mesures étant de $10 \mathrm{~min}$. Nous nous sommes placés à une période de $22.5 \mathrm{~min}$ pour cette deuxième période, qui peut donc apparaitre soit comme une harmonique pour la période de 45 min soit comme une valeur propre de l'oscillation. A partir d'une fenêtre glissante de $24 \mathrm{~h} 00$ (qui correspond à un nombre entier de périodes de ces deux seiches), nous avons calculé l'évolution temporelle de l'amplitude de l'oscillation de la surface pour ces deux périodes. Nous supposons donc implicitement que la durée caractéristique de ces seiches est supérieure à la journée.

Nous avons identifié des valeurs maximales, mais qui correspondent à des amplitudes restant faibles, puisque de l'ordre de 2 centimètres. L'exemple du 19 décembre 2016 (jour 1449) est présenté sur la figure 5. La seiche apparait comme forcée par les conditions de fort vent d'est relevées les 19 et 20 décembre. Pour T=45min, l'amplitude de la seiche est du même ordre pour les stations Ifremer, Tamaris et St Louis, ce qui correspond aux observations de MILLOT et al. (1981) puisque l'amplitude est maximale sur la frontière Ouest pour ce mode. Aucun signal n'est par contre observé sur la station du Lazaret, plus excentrée. Pour $\mathrm{T}=22.5 \mathrm{~min}$, la station de St Louis ne détecte aucun signal, ce qui est cohérent avec la seiche considérée, présentant une ligne nodale perpendiculaire à la jetée. Un signal fort y est par ailleurs observé quelques jours avant, en l'absence de signal des autres stations. Cette station est dans la grande rade, donc peut-être différemment réactive aux variations des conditions de vent, cet aspect sera à étudier. L'absence de signal pour la station de Lazaret le 19 décembre est surprenante, la position de la station étant a priori dans une zone où l'amplitude du mode $\mathrm{T}=22.5 \mathrm{~min}$ doit être importante car au voisinage d'un ventre. Notons enfin que ces résultats pour $\mathrm{T}=22.5 \mathrm{~min}$ nécessitent des études complémentaires pour être 


\section{XVèmes Journées Nationales Génie Côtier - Génie Civil \\ La Rochelle, 29 au 31 mai 2018}

confirmés, la période considérée étant très proche des plus petites périodes théoriquement observables, $\mathrm{T}=20 \mathrm{~min}$, pour la période de mesure des stations.
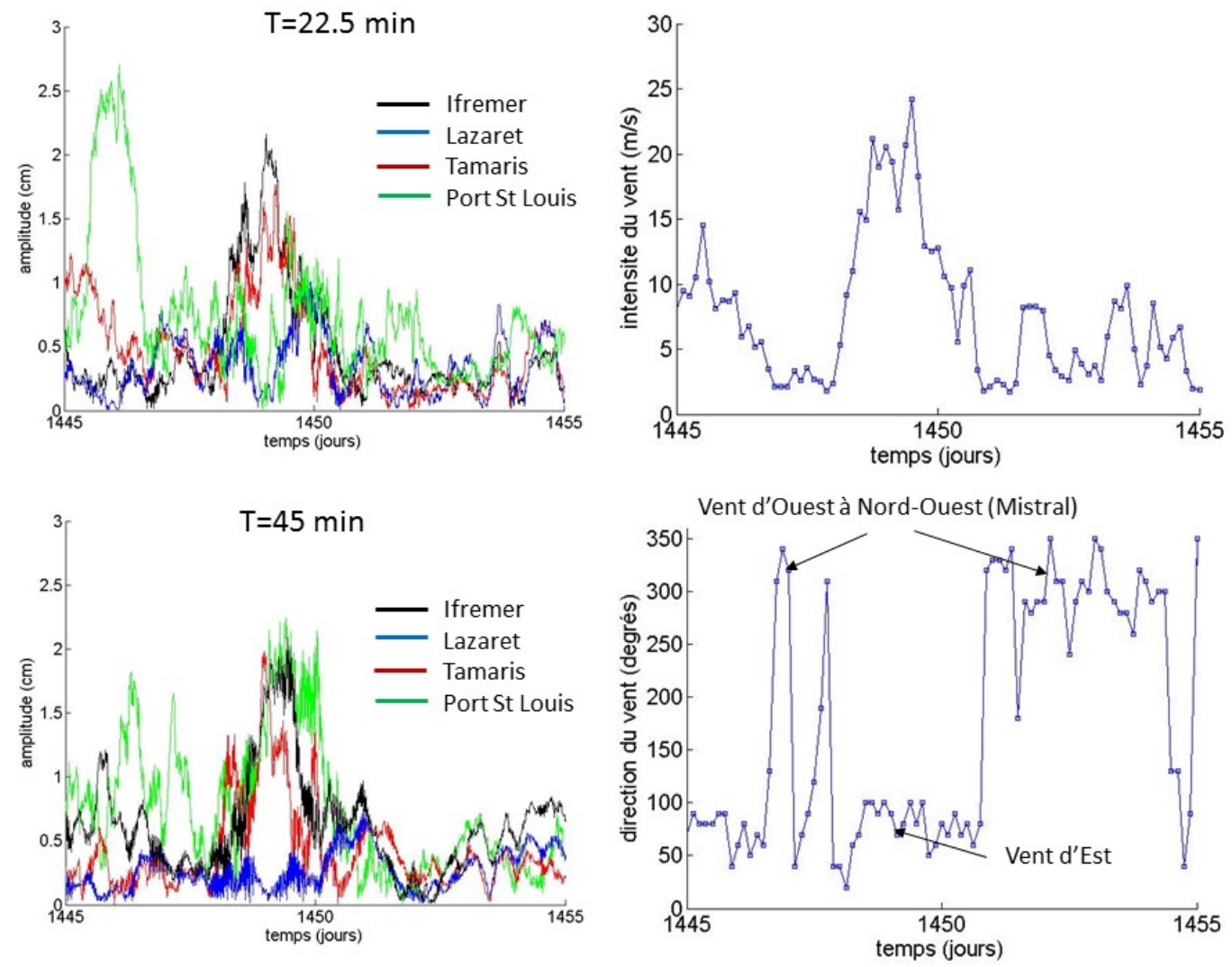

Figure 5. Analyse des seiches entre le 15 et le 24 décembre 2016.

\section{Analyse des variations de température}

Les températures mesurées par les capteurs immergés sont un indicateur pertinent des mouvements des masses d'eaux. On peut voir sur la figure 2 d'une part la variation annuelle de température, et d'autre part des chutes de température brutales en été, qui seront discutées ci-après. Les variations diurnes observées sont dues aux variations de températures de surface liées à l'ensoleillement diurne, elles sont également en partie dues à l'exposition de l'instrument qui réchauffe sans doute l'eau au niveau du capteur (situé sur la partie inférieure du tube vertical cylindrique en aluminium).

\subsection{Remontées d'eaux froides en été}

On a représenté sur la figure 6 les histogrammes des températures relevées entre le 21 juin et le 21 septembre 2017 sur les 9 stations du réseau. L'été 2017 a été marqué par un nombre important d'épisodes de Mistral qui se traduisent par des remontées d'eaux froides dont les intensités et la durée dépendent des localisations. 


\section{Thème 7 - Risques côtiers}
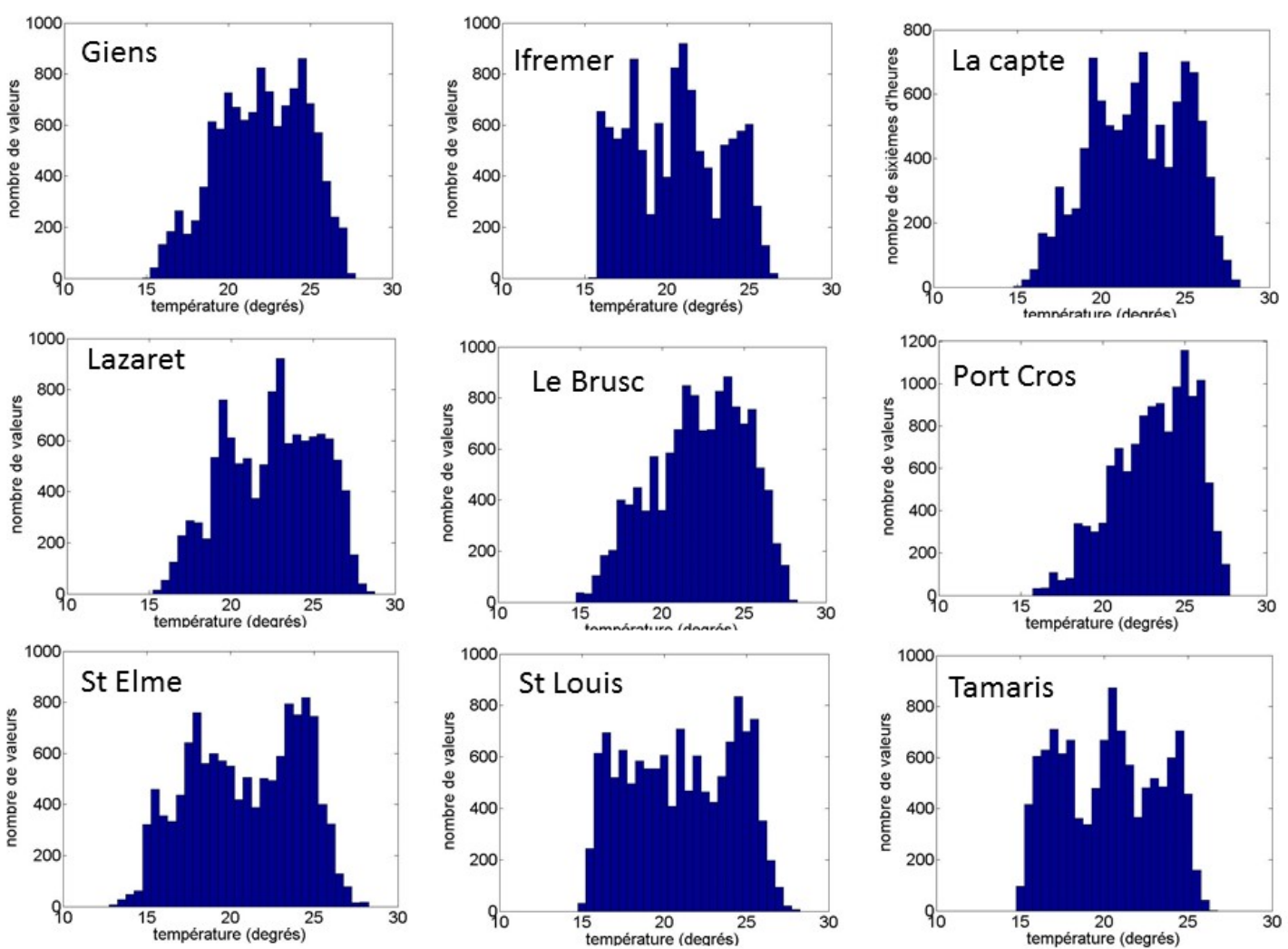

Figure 6. Histogramme des températures, été 2017.

On voit par exemple que les températures au niveau de Port-Cros, le Brusc et Giens restent globalement les plus chaudes. Ceci peut s'expliquer pour Port Cros par sa situation la plus à l'est (le Mistral n'atteint pas systématiquement cette zone) et la plus éloignée de la côte. Les sites du Brusc et de Giens sont dans des baies orientées vers l'ouest. La masse d'eau superficielle chaude est repoussée et maintenue dans la baie par le Mistral : l'apparition d'eau superficielle froide est donc retardée, et résulte plutôt du mélange issu des vagues ainsi, potentiellement, que de de l'entrainement des eaux d'un upwelling proche. La station de St Elme, et celles dans la rade de Toulon (Ifremer, Tamaris, Lazaret et St Louis) dont les orientations correspondent aux conditions d'upwelling sont fortement impactées, avec une forte proportion de températures entre 15 et $20^{\circ} \mathrm{C}$. Notons que ces épisodes d'upwellings permettent un renouvellement des eaux, notamment dans la petite rade de Toulon (DUFRESNE, 2014, DUFRESNE et al, 2014).

\subsection{Dynamique en période hivernale}

On a représenté sur la figure 7 les données de températures de l'eau à Ifremer et à Port Cros durant l'hiver 2014-2015. On observe une température mesurée à Ifremer inférieure à celle de Port Cros. On observe également une tendance à la baisse des 


\section{XVèmes Journées Nationales Génie Côtier - Génie Civil \\ La Rochelle, 29 au 31 mai 2018}

températures, ce qui est logique en cette saison. Il est intéressant de voir que la température dans la rade remonte à la fin janvier pour atteindre la même température qu'à Port Cros. Cette remontée de la température correspond à un renouvellement de la masse d'eau dans la petite rade (DUFRESNE, 2014 ; DUFRESNE et al., 2014) lié aux conditions de Mistral (station MétéoFrance de Toulon montrant la présence d'un Mistral fort fin janvier), comme en été : de l'eau du large, qui est à $\sim 13^{\circ} \mathrm{C}$ en cette saison, vient remplacer l'eau de la rade qui a pu être refroidie, en raison de la faible profondeur.

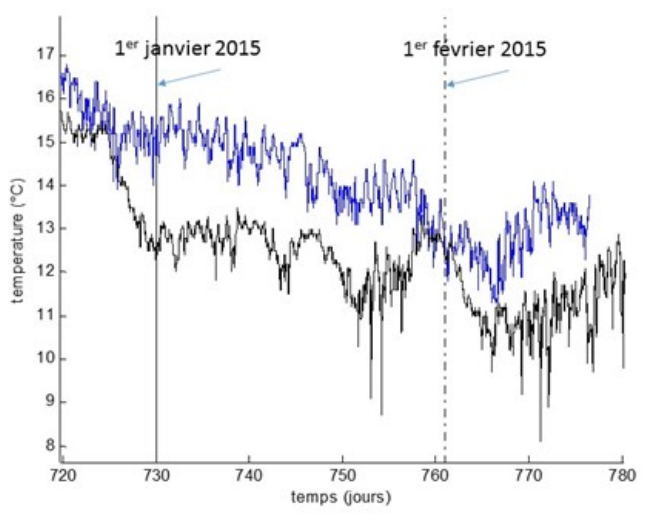

Figure 7. Evolution des températures, Ifremer (en noir) et Port-Cros (en bleu), hiver 2014-2015.

\section{Discussions et perspectives}

Nous avons montré par ces quelques exemples et illustrations les potentiels d'études et de compréhension de la dynamique littorale et côtière apportés par un réseau de capteurs de niveau d'eau et de température tel le réseau HTM-NET. Tous les résultats présentés ici ne sont pas encore pleinement exploités, comme l'étude des effets des dépressions et des conditions locales de vent sur le niveau en fonction de l'orientation des baies. Concernant la circulation associée aux upwellings, les séries temporelles de températures aux différentes stations doivent contribuer à mieux comprendre les régimes de courant associés aux diverses conditions de vent, en particulier de la présence ou de l'absence d'un vent d'ouest jusqu'à l'est des îles d'Hyères. Nous avons vu que la périodicité des mesures de 10 minutes, due initialement à des raisons techniques, ne permet pas d'étudier les oscillations de trop faible période. Le passage à une période d'acquisition de 2 minutes est actuellement testé sur la station de Saint Louis et doit être généralisé à l'ensemble des stations. L'extension du réseau vers l'ouest et au-delà de Marseille est également en cours, motivée par les activités du M.I.O. sur cette frange littorale. Ces données ont aussi pour finalité d'être utilisées pour alimenter des programmes de circulation à l'échelle côtière, dont la résolution de plus 


\section{Thème 7 - Risques côtiers}

en plus fine permet de mieux représenter la zone littorale. Outre l'intérêt en océanographie côtière et littorale, les mesures et modélisations associées peuvent être un outil pertinent pour les études de transport de matière (organismes, sédiments, polluants, etc...), d'impacts associés aux événements climatiques (vent, houles et surcotes), problématiques présentant un intérêt socio-économique important (données de niveau d'eau, qualité des eaux, risques naturels et anthropiques, impacts sur le littoral).

\section{Remerciements}

Le programme d'observation HTM-NET a bénéficié d'un programme spécifiquement dédié au littoral de l'agglomération toulonnaise, "Hydrodynamique et Transport de MES : Niveaux d'Eau et Température, HTM-NET » puis des programmes OLBIA et CAPTILE co-financés par la communauté d'agglomération Toulon Provence Méditerranée (TPM) et de co-financements récurrents depuis 2013 de la part du CNRS/INSU, dans le cadre du programme national "Systèmes d'Observations et d'Expérimentation pour la Recherche en Environnement » sur le long terme (SOERE) puis SOERIL, Littoral -Trait de Côte. Le Parc National de Port Cros, la Métropole TPM et la Ville de Hyères sont remerciés pour les autorisations d'implantation des stations, le Shom pour sa collaboration et MétéoFrance pour les données météorologiques.

\section{Références}

DUFRESNE C. (2014). Compréhension et analyse des processus hydro-sédimentaires de la baie de Toulon. Apport à la modélisation de la dispersion des radionucléides. Thèse de l'Université de Toulon, novembre 2014.

DUFRESNE C., DUFFA C., REY V. (2014). Wind forced circulation in the Bay of Toulon and water exchanges at the Little Bay fairway. Ocean Dynamics, Vol. 64(2), pp 209-224. https://doi.org/10.1007/s10236-013-0676-3

GUIHOU K. (2013). Étude de la dynamique du Courant Nord au large de Toulon, à l'aide de modèle, observations in-situ et données satellites. Thèse de l'Université de Toulon, décembre 2013.

MILLOT C. (1979). Wind induced upwellings in the Gulf of Lions. Oceanologica Acta, Vol. 2(3), pp 261-274.

MILLOT C., BROYARD R., METAIS O., TINE J. (1981). Les oscillations propres de la Rade de Toulon. Oceanologica Acta, Vol. 4(3), pp 259-262.

TAUPIER-LETAGE I., PIAZZOLA J., ZAKARDJIAN B. (2013). Les îles d'Hyères dans le système de circulation marine et atmosphérique de la Méditerranée. Sci. Rep. Port-Cros natl Park, Vol. 27, pp 29-52. 